\title{
A simple hair control approach during intra-oral surgical procedures
}

\author{
Shruti Chhabra, Yuvika Raj Kumar, Naveen Chhabra \\ D.A.V. (C) Dental College, Model Town, Yamuna Nagar, Haryana
}

doi: 10.3396/ijic.V7i3.023.11

\begin{abstract}
While the majority of post-operative infections originate from endogenous flora, patient factors can also contribute to these infections. Facial hair around the operative field, especially hair of a beard, can be a cause for reduced access to the operative field and post-operative infection. The solution to this problem is either removal of the hair or the application of various hair control techniques. Pre-operative hair removal has been demonstrated to actually increase the risk of post-operative infection so hair control techniques should be chosen wherever conditions permit. This technical note explains a very simple, economical and less time consuming method for controlling hair around the oral cavity, which involves the use of a face mask that can be offered to patients who are not willing to get their hair removed. This may be important due to their religious beliefs especially patients from the Sikh and Muslim community and also patients who cannot bear the increased cost of treatment due to application of some expensive hair control methods.
\end{abstract}

\section{Key words}

Face mask; Beard; Infection Control, Hair control techniques

Surgical site infections (SSIs) are defined as infections occurring within 30 days after a surgical operation or within one year after the placement of an implant. A surgical site infection can occur in either the incision or deep tissue at the operation site. ${ }^{1}$ Preventing disease transmission is the primary goal of infection control. Because of the nature of dental care, patients and Dental Health Care Personnel (DHCP) have the potential for exposure to a variety of micro-organisms in dental health care settings. In most SSIs, the causative pathogens arise from the patient's endogenous flora, most commonly being Staphylococcus aureus,

\footnotetext{
Corresponding author

Yuvika Raj Kumar

Genesis Institute of Dental Sciences \& Research, Ferozepur, Punjab

Telephone No: 09592255552

Email: dryuvikarajkumar@yahoo.com
} 
coagulase-negative staphylococci, Enterococcus species and Escherichia coli. The pathogens responsible for SSIs also depend on the nature of the procedure, for example, in head and neck surgery, $S$. aureus, streptococci and oropharyngeal anaerobes (e.g. peptostreptococci) are more common. ${ }^{2}$ The other source for origin of SSIs pathogens can be from exogenous sources like members of the surgical team, operating theatre environment, and instruments and materials brought within the sterile field during the procedure.

Since the days of Lister and Semmelweis, skin hair was believed to be a source of contamination. More recent studies have confirmed their suspicions. Summers et al have noted that $50 \%$ of medical staff and $60 \%$ of inpatients carried pathogenic bacteria in their hair. Staphylococcus aureus was the most common organism noted. E. coli, S. viridians and Pseudomonas were also present. Most of the $S$. aureus from the hospital personnel was resistant to commonly used antibiotics. The patients whose hair carried pathogenic bacteria had wound infections more commonly than the non carriers. ${ }^{3}$

Several intra-operative hair control techniques have been identified which include the following: shaving, clipping, depilatory cream, gelling, braiding, banding or clasping with haemorrhoid bands, ligatures, metal foil or urinary catheters, the use of chlorhexidine, covering the hair with a swimming cap, adhesive drapes and/or dressings. ${ }^{4}$

Shaving of hair carries an infection rate $3.1 \%$ when done just before surgery, $7.1 \%$ when done up to 24 hours before surgery and 20\% when done more than 24 hours before surgery. Removal of hair with electric clippers carries an infection rate of $1.8 \%$. The lowest infection rate $(0.6 \%)$ is associated with no attempt at hair removal or use of a depilatory cream. ${ }^{3}$ According to a study done by Alexander JW et al razor shaving of a site is unnecessary and increases the risk of post-operative infection. ${ }^{5}$ The Centre for Disease Control and Prevention (CDC) recommended guidelines for prevention of SSIs indicate avoidance of hair removal around the operation site, unless it will interfere with the procedure. If hair is to be removed, then it should be done immediately before the operation, preferably with clippers. ${ }^{6}$
The haemorrhoid bands are used by mounting on to an artery clip that grasps the hair section after twisting and folding over, while the band is rolled down over the clip. ${ }^{6}$ Use of haemorrhoid bands can also be done for controlling hair in this region but still the hair remains exposed and the reduction in bacterial load is less. Metal foil can also be used to tie hair, but it falls off very quickly. Use of loads of chlorhexidine is another method to control hair, but it causes stickiness and staining. Application of an adhesive drape can also be done to keep the hair of the beard and moustaches away, but its removal is uncomfortable in a patient undergoing local anaesthetic procedure. One more method of hair control is with the use of staples, ${ }^{7}$ commonly employed for skin closure. Hair is made wet and staples are applied to hold it away from the incision line. It is a quick and easy technique, but is best suited to thick scalp skin rather than thin facial skin. ${ }^{6}$

It is emphasized in literature that it is important to reduce shedding of hair in the operating room, which is best achieved by covering the hair. When hair is covered, it is prevented from obscuring the operative field or becoming entangled in sutures during the procedure and during wound closure.

The distribution of hair on the face generally does not pose a problem while working in the oral cavity in the majority of patients. The exception is patients who have long beards and moustaches (Fig 1). These hairs being in close proximity to the working area can masquerade a problem to the maxillofacial surgeon during surgical procedures in and around this area.

The hair can obscure the operative field, which reduces the access to the operative site. Entangling of hair in sutures or the wound during closure can incite foreign body reactions and granulomas. Hair entangled in the wound can also be a cause for infection as it acts as a foreign body. As asepsis and access to the operative site are one of the prime requirements of surgical procedures, methods should be employed to either remove the hair or keep the hair away from the operative field.

The reasons for having a beard are varied. Requiring the beard to be shaven off can have aesthetic, communal, 
status, religious, employment consequences and raise vindicated objections from the patient. The majority of the patients in this study are from the Sikh and Muslim community, who are adamant believers of their religion. Under no circumstances will these patients consent to get the hair removed. So, with the presence of hair around the oral cavity, it becomes difficult to maintain asepsis and achieve good access to the surgical site for these patients. One option is to apply a disinfectant to the operative field, but this does not solve the problem of access to the oral cavity. Additionally the disinfectant can temporary discolour the beard.

During a review of the literature, the use of a face mask to keep the hair away in the cases of a sternotomy procedure was noted by Sanders LH et al. ${ }^{8}$

To deal with the problems of facial hair, this technical note mentioned the application of a face mask to the area of beards and moustaches and the use of a cheek retractor. It involves the positioning of the beard inferiorly and posteriorly with the help of a face mask, in a way that almost all the hairs are covered by the

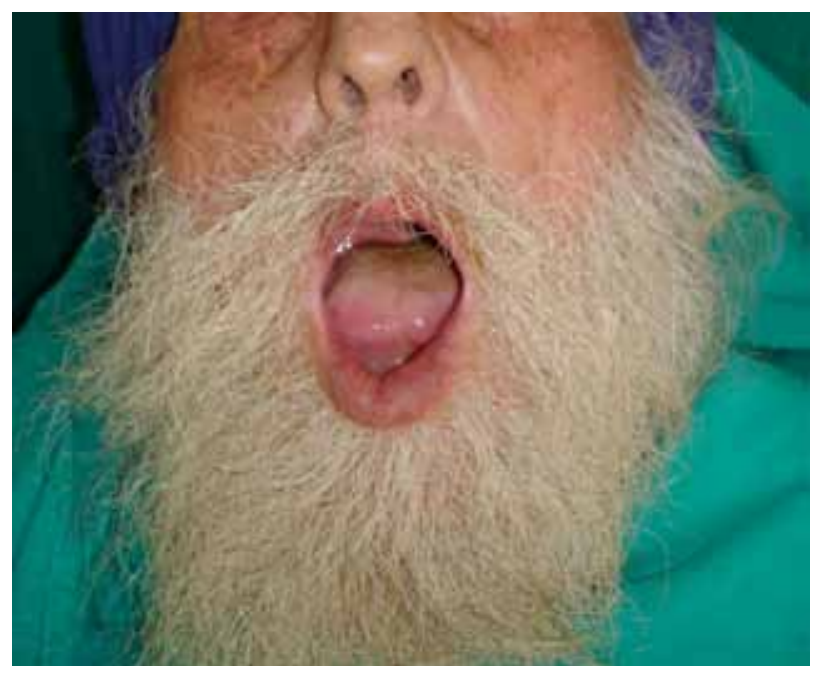

Figure 1: Excessive facial hair (beard) around the operative field impeding access and being a source of infection mask and then the mask is tied at the back of head (Fig 2). If the hair of the moustaches pose obstruction to the operative field, then an additional face mask can be used in this region in a similar manner to keep the hair away (Fig 2). In addition to the application of masks, the use of a cheek retractor can also be used to further aid in improving access to the field (Fig 2). In this way, the hair will be kept away with the mask and the cheeks and lips with the retractor, thus increasing the visual access to the operative field. This technique helps to reduce the problem of hair interfering during procedures in the oral cavity, which improves site access, and decreases the duration of surgery, postoperative complications and risk of infection.

This is a very simple and economical solution for managing hair around the oral cavity, where hair removal is avoided and is in accordance with the CDC guidelines. With the application of this technique, it is possible to respect the patient's beliefs and values regarding their religion and way of life. This may be of prime importance for specific individuals and a way to accommodate them during oral surgical procedures.

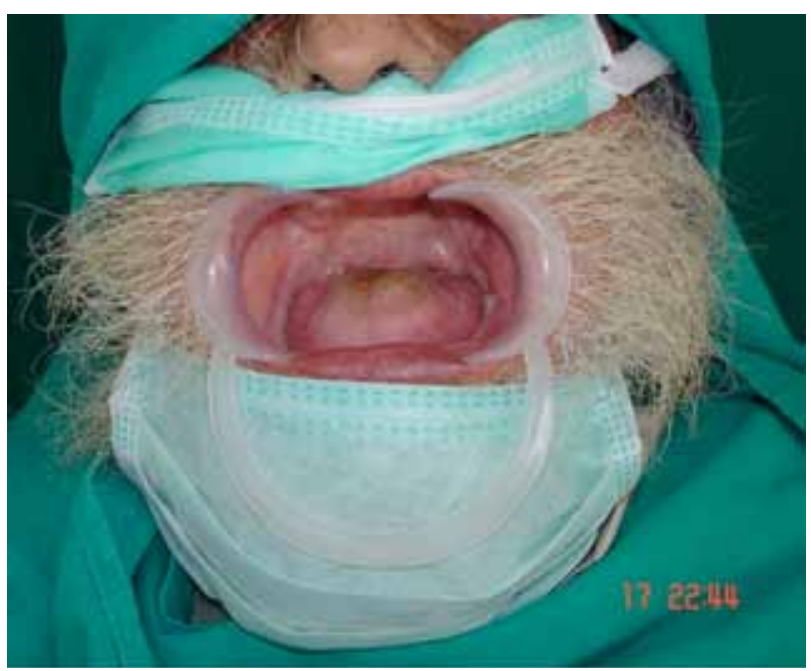

Figure 2: Increased access to the operative field with aid of masks and cheek retractor 


\section{References}

1. Managram AJ, Horan TC, Pearson ML, Silver LC, Jarvis WR. Hospital infection control practice advisory committee: Guideline for prevention of surgical site infection, 1999. Infection Control \& Hospital Epidemiology 1999; 20: $247-$ 278.

2. Owens CD, Stoessel K. Surgical site infections: epidemiology, microbiology and prevention. Journal of Hospital Infection 2008; 70 (S2): 3-10.

http.dx.doi.org/10.1016/S0195-6701(08)60017-1

3. Edlich RF, Jackson EM, Neal JG, Kron IL. A scientific basis for choosing the technique of hair removal used prior to wound closure. Journal of Emergency Nursing 2000; 26: 134-139. http.dx.doi.org/10.1016/S0099-1767(00)90056-4

4. Carter LM, Wales CJ. Hair control: How to get ahead in maxillofacial surgery. Br Journal of Oral Maxillofacial Surgery 2007; 45: http.dx.doi.org/10.1016/j.bjoms.2007.07.152

5. Alexander JW, Fischer JE, Boyajian M, Palmquist J, Morris MJ. The influence of hair-removal methods on wound infections. Arch Surg 1983; 118(3): 347-352.

6. O'Neill JK. A novel method of hair control during face lift surgery: haemorrhoid bands. British Journal of Plastic Surgery 2005; 58: http.dx.doi.org/10.1016/j.bjps.2005.02.008

7. O'Neill JK, Lee S. Controlling hair during craniofacial surgery requiring scalp incisions. Journal of Plastic, Reconstructive \& Aesthetic Surger. 2009;

http.dx.doi.org/10.1016/j.bjps.2009.08.004

8. Sanders LH, Woost FJ, Meer AD, Giebelen DJ, Weightman WM. Sternotomy and the beard. Heart, Lung and Circulation 2008; 17: 139-140.

http.dx.doi.org/10.1016/j.hlc.2007.09.005 